\title{
Extracción y Caracterización Fisicoquímica del Aceite de la Semilla (Almendra) del Marañón (Anacardium occidentale $L$ )
}

\author{
Jennifer J. Lafont ${ }^{(1)^{*}}$, Manuel S.Páez ${ }^{(1)}$ y Alfonso A. Portacio ${ }^{(2)}$ \\ (1) Universidad de Córdoba, Facultad de Ciencias Básicas e Ingeniería, Departamento de \\ Química, (2) Departamento de Física, Carrera 6 No 76-103. Km 3, vía Cereté. Córdoba-Colombia \\ (e-mail: jenniferlafontm@yahoo.es) \\ ${ }^{*}$ autor a quien debe ser dirigida la correspondencia
}

Recibido Feb. 26, 2010; Aceptado Abr. 13, 2010; Versión Final recibida May. 04, 2010

\begin{abstract}
Resumen
El objetivo principal del trabajo que se presenta en este artículo es la evaluación de diferentes métodos de extracción de aceite de semillas de almendras (Anacardium occidentale $L$ ) y caracterización de sus propiedades físico-químicas. El procedimiento consiste en someter la muestra a procesos de extracción mecánica o prensado y a extracción con solventes aplicando dos métodos: inmersión e inmersión-percolación. El método más eficiente fue el de inmersiónpercolación usando n-hexano como solvente. El rendimiento obtenido es del $(97.78 \pm 1.32) \%$. El análisis de ácidos grasos por HPLC reveló un alto contenido de ácido oleico (61.36\%). De estos resultados se recomienda la extracción con solventes para la industria de jabones, cosméticos y la extracción con prensado para la industria alimenticia.
\end{abstract}

Palabras claves: almendras, anacardium occidentale L., extracción, caracterización fisicoquímica, perfil de ácidos grasos

\section{Extraction and Physicochemical Characterization of Seed Oil (Almond) of Cashew nut (Anacardium occidentale $L$ )}

\begin{abstract}
The main objective of the work presented in this paper is the evaluation of different methods for the extraction of almond oil (Anacardium occidentale $L$ ) and the characterization of its physicochemical properties. The procedure involves subjecting the sample to mechanical extraction process, or pressing and solvent extraction using two methods, immersion and immersion-percolation. The most efficient method was the immersion-percolation using $n$-hexane as solvent. The yield obtained is $(97.78 \pm 1.12) \%$. The fatty acid analysis by HPLC revealed high content of oleic acid (61.36\%). From these results solvent extraction is recommended for the soap industry, for cosmetics and extraction by pressing in the food industry.
\end{abstract}

Keywords: cashew nut, anacardium occidentale L., extraction, physicochemical characterization, fatty acid profile 


\section{INTRODUCCIÓN}

El marañón (Anacardium occidentale $L$ ), es un árbol silvestre que pertenece a la familia Anacardiaceae, originario de las zonas tropicales de América y del noroeste brasileño que presenta excelentes propiedades medicinales y nutricionales (Ofusori et al., 2008); posee ramificación baja y altura media variable de 5 a 14 metros, dependiendo del genotipo y condición climática, su fruto puede ser rojo y amarillo, es astringente y nutricional, contiene $47 \%$ de grasa, $21 \%$ de proteína y $22 \%$ de carbohidratos, vitaminas y aminoácidos (Mestres et al, 2009; Omosuli et al.,2009) el fruto está unido a un pseudo fruto o nuez de color gris y forma arriñonada con una composición química del $46.3 \%$ de aceite respecto a su peso total (Castro et al., 2007). Este árbol es ampliamente aprovechado: El tronco se utiliza para la construcción de muebles; la corteza para la elaboración de tintas; la manzana o fruto se usa en la producción de dulces, conservas, vinos; es antiescorbútico y diurético; la nuez, se emplea para endurecer chocolates y en la fabricación de dulces y jugos, (Chipojola et al.,2009). En la nuez se producen dos aceites: uno de la cáscara (pericarpio, testa), de color negro, viscoso, irritante y cáustico que contiene compuestos fenólicos (Setianto et al., 2009), cardol con aplicaciones en la industria de plásticos, insecticidas y tintas; el otro aceite es extraído de la almendra, es de color amarillo brillante y menos viscoso que el aceite anterior, siendo el objeto de este estudio.

A pesar de la gran aplicabilidad de todas las partes de la planta del marañón, en la actualidad no se han reportado estudios sobre las características fisicoquímicas del aceite de la almendra; desde este punto de vista, este trabajo pretende aportar nuevos datos que sirvan como base para futuras investigaciones. El objetivo de este trabajo consiste en evaluar los métodos de extracción y las características fisicoquímicas del aceite de la almendra del marañón (Anacardium occidentale $L$ ), mediante la determinación de sus propiedades fisicoquímicas y el perfil de ácidos grasos.

\section{METODOLOGÍA}

La metodología inicia con la recolección de nueces en frutos maduros del marañón (Anacardium occidentale $L$ ), en los municipios de: Chinú - departamento de Córdoba y el Roble - departamento de Sucre (Colombia); posteriormente fueron seleccionadas las de mejor estado y secadas al sol durante tres días para disminuir la irritabilidad del aceite contenido en la cáscara, facilitando así la extracción manual de la almendra, haciéndose necesaria la utilización de guantes. La extracción del aceite de la almendra del marañón se realizó mediante dos métodos convencionales: prensado y extracción con disolventes; en ambos casos la almendra fue triturada utilizando mortero cerámico hasta formar una masa fina, convirtiéndose en la muestra de estudio. En la extracción por prensado se pesaron $100 \mathrm{~g}$ de muestra, en una balanza analítica OHAUS de \pm $1 \times 10^{-4} \mathrm{~g}$ de precisión; la almendra triturada fue calentada y prensada utilizando una prensa hidráulica con cilindro de superficie lisa donde se introduce la muestra y un pistón al que se le aplica una fuerza de compresión de 10 Ton, el aceite extraído de esta operación se recogió en un beaker, la torta que quedó en el cilindro se volvió a prensar en las mismas condiciones para obtener mayor cantidad de aceite (Hernández et al, 2007). Este procedimiento se realizó por triplicado; con el propósito de evaluar el valor promedio $(\bar{X})$, la desviación estándar $(\sigma)$ y calcular el rendimiento del método dividiendo la masa total del aceite entre la cantidad de muestra de almendra utilizada. La extracción por disolvente se realizó de dos maneras: por inmersión o reflujo sencillo y percolación-inmersión o reflujo con soxhlet; utilizando tres solventes diferentes para cada caso: hexano (Merck; 99.0\%), acetato de etilo (Merck, 99.5\%) y éter de petróleo (Panreac, $99.0 \%$ ); siguiendo la metodología propuesta por (Trevejo y Mauri, 2002). Cada solvente se trabajó a sus temperaturas respectivas de ebullición así: hexano $\left(67^{\circ} \mathrm{C}\right)$, acetato de etilo $\left(45^{\circ} \mathrm{C}\right)$ y éter de petróleo $\left(57^{\circ} \mathrm{C}\right)$; usando $100 \mathrm{~g}$ de muestra. El aceite obtenido por ambos procedimientos, se sometió a un proceso de desgomado con el fin de separar algunas impurezas presentes como gomas, fosfolípidos y fosfátidos; para lo cual, el aceite se somete a calentamiento a $90^{\circ} \mathrm{C}$, luego se le adiciona el $10 \%$ de agua destilada y se deja reposar por 30 minutos para llevarla a centrifugación, (Castro et al, 2007). Este procedimiento para ambas extracciones se realiza por triplicado. Para determinar las propiedades físicas, a los aceites extraídos por prensado y solvente, se les midieron los parámetros de Densidad (Páez et al., 2008) y Viscosidad (Páez et al., 2009), a las temperaturas de $(283.15,288.15,293.15,298.15,303.15$ y 308.15$) \mathrm{K}$. La densidad 
fue determinada con el picnómetro de Wood-Brusie de cuello capilar. El procedimiento inicia con la calibración del picnómetro que consiste en pesarlo vacío, llenarlo con agua y colocarlo en el termostato a temperatura controlada, dejándolo en un baño por 30 minutos para alcanzar el equilibrio térmico, luego se lleva a la marca de aforo y se vuelve a pesar a temperatura ambiente de esta manera se calcula el volumen del picnómetro; este procedimiento se repite pero utilizando aceite para la determinación de la densidad. La viscosidad, se midió con un viscosímetro de Ubbelohde de nivel suspendido, la calibración se hace con agua bidestilada a las 6 temperaturas descritas con un índice de repetitividad de 10 veces. El mismo procedimiento se realizó con el aceite.

Para determinar las propiedades químicas, a los aceites extraídos por prensado y solvente se realizaron: El análisis de humedad y materia volátil siguiendo la metodología propuesta por (Cenzano, 1994); en el cual se toma una capsula de porcelana completamente seca y esterilizada, se pesan 10 gramos de aceite y se calientan a $105^{\circ} \mathrm{C}$ durante $30 \mathrm{~min}$, se enfría y se pesa. El procedimiento se repite hasta que la diferencia entre las dos pesadas no exceda el $0.05 \%$. Los índices de acidez (AOCS Ca 5a - 40., 1997); peróxidos (AOCS Cd 8-53., 2003), yodo (AOCS Cd 1-25., 1997) y saponificación (AOCS Cd 3-25., 2003), se evaluaron de acuerdo con la metodología de la American Oil Chemists Society. Todos los análisis se realizaron por triplicado, de la siguiente forma: En el índice de acidez, se pesan $5 \mathrm{~g}$ de aceite y se adiciona una solución alcohólica que se titula con hidróxido de sodio. En el índice de acidez se expresan los ácidos grasos libres como acido oleico. En el índice de peróxido, se pesan 5 gramos de aceite y se diluye en una solución de ácido acético-cloroformo 3:2; se agrega yoduro de potasio por 1 minuto, y se adiciona agua, luego se titula con tiosulfato de sodio $0.1 \mathrm{~N}$, hasta que el color amarillo desaparezca. En el índice de yodo, se pesan 5 gramos de aceite y se disuelve en tetracloruro de carbono, luego se le agregan $25 \mathrm{ml}$ del reactivo de Wijs, dejando reposar la solución en la oscuridad, se le agrega solución de yoduro de potasio y se procede a titular, luego se adiciona almidón y se continua titulando hasta que el color azul desaparezca. En el índice de saponificación, se pesan 5 gramos de aceite y se agrega una solución alcohólica de hidróxido de potasio, se prepara un blanco simultáneamente, se colocan el blanco y la muestra en el condensador hasta completar la saponificación (1 hora), luego la muestra se titula con solución de acido clorhídrico. Todas las propiedades químicas se realizan por triplicado. El perfil de ácidos grasos se realizó siguiendo la metodología de la American Oil Chemists Society (AOCS Ce 1C-89., 1997) utilizando cromatografía líquida de alta resolución (HPLC), con detector FID, Columna capilar SP-2340 (78\% de cianopropilmetilsilosano - alta polaridad) con 60 metros de largo, $0.25 \mathrm{~mm}$ de diámetro interior y el espesor de la película de 0.25 $\mu \mathrm{m}$. Este ensayo se realizó por duplicado.

\section{RESULTADOS Y DISCUSIÓN}

En la tabla 1, se reporta el valor promedio del rendimiento y la desviación estándar en porcentaje $\overline{(X} \pm \sigma) \%$ para la extracción del aceite de la almendra de marañón. Del análisis de estos resultados, es evidente que: El valor del rendimiento obtenido en la extracción por el método con prensado es del $(68.12 \pm 0.45) \%$ lo que indica una eficiencia pobre, eso es debido tal vez, a que en la torta queda retenida parte del aceite, por lo cual recomendamos su aprovechamiento como subproducto a nivel industrial.

Es evidente que la extracción por el método de reflujo sencillo o inmersión presenta un mayor rendimiento usando hexano como solvente, el cual es del $(77.88 \pm 1.57) \%$, esto tal vez se debe a que el hexano al no poseer grupos funcionales en su estructura química (como ocurre con el éter y el ester) facilite la liberación y recuperación del aceite durante el proceso. Vale la pena destacar que las características organolépticas del aceite obtenido con hexano presenta un color amarillo transparente y brillante, e innodoro; mientras que el rendimiento y las características del obtenido con el éter de petróleo es del $(60.49 \pm 0.78) \%$ con un color amarillo opaco y un olor muy desagradable, lo cual tal vez se debe a su menor recuperación respecto al método anterior; de igual forma ocurre con el aceite obtenido usando como solvente acetato de etilo que posee un color rojizo y olor desagradable. 
Tambien es evidente de la tabla 1, que el más alto rendimiento para la extracción por el método de soxhlet se obtiene usando hexano, ya que su rendimiento es del $(97.78 \pm 1.32) \%$, lo cual evidencia la alta eficiencia de este solvente.

Tabla 1: Porcentajes de aceite de almendra de marañón extraídos por ambos métodos

\begin{tabular}{lcc}
\hline \multicolumn{1}{c}{ Método } & Solvente & $\begin{array}{c}\text { Rendimiento } \\
\overline{(X \pm \sigma) \%}\end{array}$ \\
\hline Prensado & & $68.12 \pm 0.45$ \\
\hline Solvente con inmersión & Éter de Petróleo & $60.49 \pm 0.78$ \\
(Reflujo Sencillo) & Acetato de etilo & $41.40 \pm 1.36$ \\
& Hexano & $77.88 \pm 1.57$ \\
\hline Solvente con inmersión -Percolación & Éter de Petróleo & $86.13 \pm 1.12$ \\
(Reflujo por Soxhlet) & Acetato de etilo & $55.73 \pm 1.25$ \\
& Hexano & $97.78 \pm 1.32$ \\
\hline
\end{tabular}

En la tabla 2, se reportan el valor promedio y la desviación estándar $\overline{(X} \pm \sigma) \%$ de la densidad y la viscosidad del aceite de la almendra de marañón extraído por prensado y soxhlet a diferentes temperaturas. De los resultados presentados en la tabla 2, es evidente que el aceite obtenido por el método con prensado está en buen acuerdo con los valores establecidos para densidad de aceites virgenes. Así, la CODEX establece para aceites virgenes (aceite de oliva) un valor para la densidad con un rango de 0.9 a $0.96 \mathrm{~g} / \mathrm{mL}$ a $20^{\circ} \mathrm{C}(293.15 \mathrm{~K})$; mientras que la FAO y OMS (Organización mundial de la salud) establecen un rango de 0.910-0.916 g/mL, para este mismo aceite. El valor mas bajo obtenido para la densidad usando el método soxhlet a cada temperatura estudiada probablemente se debe a la presencia de trazas de solvente en el aceite, situándolos por debajo de la norma establecidad por CODEX, FAO y OMS. Mientras que los resultados obtenidos en viscosidad cinemática a $20^{\circ} \mathrm{C}(293.15 \mathrm{~K})$ para el aceite extraído por prensado es de $(86.48 \pm 0.05) \mathrm{m}^{2} / \mathrm{s}$, y para el extraído con solvente es de $(45.60 \pm 0.02) \mathrm{m}^{2} / \mathrm{s}$ a la misma temperatura; se aprecia que este último resultado se reduce aproximadamente a la mitad del primero; lo cual indica que esta propiedad se ve afectada por la utilización de solventes orgánicos en la obtención del aceite; también se observa que la viscosidad del aceite extraído por ambos métodos aumenta con la disminución de la temperatura.

Tabla 2: Valores de densidad y viscosidad del aceite de almendra de marañón extraído por prensado y soxhlet a diferentes temperaturas

\begin{tabular}{ccccc}
\hline $\mathrm{T}$ & $\begin{array}{c}\text { Densidad Prensado } \\
\mathrm{K}\end{array}$ & $\begin{array}{c}\text { Densidad Soxhlet } \\
\overline{(X} \pm \sigma) \mathrm{g} / \mathrm{cm}^{3}\end{array}$ & $\begin{array}{c}\text { Viscosidad Prensado } \\
(\bar{X} \pm \sigma) \mathrm{g} / \mathrm{cm}^{3}\end{array}$ & $\begin{array}{c}\text { Viscosidad } \\
\text { Soxhlet } \\
(\bar{X} \pm \sigma) \mathrm{m}^{2} / \mathrm{s}\end{array}$ \\
\hline 283.15 & $0.9205 \pm 0.0003$ & $0.9055 \pm 0.0003$ & $141.97 \pm 0.09$ & $69.59 \pm 0.07$ \\
288.15 & $0.9171 \pm 0.0004$ & $0.9022 \pm 0.0006$ & $109.97 \pm 0.06$ & $55.78 \pm 0.09$ \\
293.15 & $0.9137 \pm 0.0002$ & $0.8988 \pm 0.0005$ & $86.48 \pm 0.05$ & $45.60 \pm 0.02$ \\
298.15 & $0.9103 \pm 0.0007$ & $0.8953 \pm 0.0005$ & $68.98 \pm 0.04$ & $37.87 \pm 0.03$ \\
303.15 & $0.9070 \pm 0.0006$ & $0.8919 \pm 0.0006$ & $53.49 \pm 0.09$ & $31.77 \pm 0.04$ \\
308.15 & $0.9034 \pm 0.0005$ & $0.8885 \pm 0.0008$ & $40.47 \pm 0.07$ & $27.23 \pm 0.03$ \\
\hline
\end{tabular}

En la tabla 3, se reportan el valor promedio y la desviación estándar $\overline{(X} \pm \sigma) \%$ de los indices de humedad, acidez, peróxido, yodo y saponificación analizados para los aceites de la almendra de marañón extraídos por prensado y soxhlet. De acuerdo con esta tabla, el porcentaje de humedad y materia volátil del aceite extraído por prensado es de $(0.17 \pm 0.01) \%$ y por soxhlet de $(5.362 \pm$ $0.006) \%$; la diferencia entre estos valores puede ser debido a la contaminación del aceite con 
trazas de solvente en el segundo caso, haciendolo de esta forma no recomendable para la industria alimenticia, mientras que el valor de humedad y materia volátil obtenido para el aceite por el método de prensado se encuentra dentro de los parámetros nacionales e internacionales establecidos para aceites de uso alimenticio; tal como lo evidencian las normas de: CODEX a $105^{\circ} \mathrm{C}$ valor máximo $0.2 \%$; y ANDI $0.1 \%$ (FAO Y OMS, 1999), reiterándose de esta forma que el proceso de pre-tratamiento o desgomado con agua no influye significativamente en la humedad del aceite, y lo hace apropiado para retirar los fosfolípidos y fosfátidos presentes.

Tabla 3: Propiedades analizadas para los aceites de almendra extraídos por prensado y soxhlet

\begin{tabular}{lcccc}
\hline \multicolumn{1}{c}{ Índices } & $\begin{array}{c}\text { Soxhlet } \\
(\bar{X} \pm \sigma)\end{array}$ & $\begin{array}{c}\text { Prensado } \\
(\bar{X} \pm \sigma)\end{array}$ & CODEX & ANDI \\
& $5.362 \pm 0.006$ & $0.17 \pm 0.01$ & 0.20 & 0.10 \\
\hline Humedad (\%) & $1.6 \pm 0.2$ & $0.06 \pm 0.01$ & 4.00 & 2.07 \\
Acidez $(\mathrm{mg} \mathrm{KOH} \mathrm{/} \mathrm{g)}$ & $1.3989 \pm 0.0006$ & $1.1 \pm 0.2$ & 15.00 & 5.00 \\
Peróxido $\left(\mathrm{meq} \mathrm{O} \mathrm{O}_{2} / \mathrm{Kg}\right)$ & $83.0 \pm 0.7$ & $85.1 \pm 0.7$ & $75-94$ & $75-94$ \\
Yodo $(\mathrm{cg} \mathrm{l} / \mathrm{g})$ & $175.4 \pm 0.4$ & $194.4 \pm 0.9$ & $184-196$ & $184-196$ \\
Saponificación $(\mathrm{mg} \mathrm{KOH} \mathrm{/} \mathrm{g)}$ & & & & \\
\hline
\end{tabular}

El índice de acidez del aceite extraído por prensado es de $0.06 \pm 0.01 \mathrm{mgKOH} / \mathrm{g}$ y por soxhlet de $1.6 \pm 0.2 \mathrm{mgKOH} / \mathrm{g}$, siendo mucho mayor el valor de esta prueba para el último método. El origen de este valor se asocia probablemente al hecho de que la humedad en el aceite produce una reacción de hidrólisis de los triglicéridos formando ácidos grasos libres; sin embargo el aceite obtenido por prensado presenta un valor bajo de acidez lo que indica que es mucho más estable con el tiempo, estando dentro de los parámetros establecidos para la industria alimenticia según la CODEX ALIMENTARIUM que deben tener un valor máximo $4 \mathrm{mg} \mathrm{KOH} / \mathrm{g}$ en el aceite(FAO Y OMS, 1999). El índice de peróxido mide la oxidación del aceite fresco o el grado de rancidez en el momento de la prueba; se considera que los productos con índice superior a $2 \mathrm{meq} \mathrm{de} \mathrm{O}_{2} / \mathrm{Kg}$ son altamente propensos a mostrar rancidez (Belén et al 2005). Los valores promedios y las desviaciones estándar $(\bar{X} \pm \sigma)$ del índice de peróxido del aceite de la almendra del marañón extraído son de $1.1 \pm 0.2$ meq de $\mathrm{O}_{2} / \mathrm{Kg}$ y $1.3989 \pm 0.0006$ meq de $\mathrm{O}_{2} / \mathrm{Kg}$ para la extracción por prensado y por soxhlet respectivamente; se observa que ambos valores están por debajo del límite máximo permitido; indicando que son bastante estables a los procesos oxidativos, se puede decir que para la industria alimenticia ambos aceites cumplen con las normas CODEX y ANDI las cuales consideran para los aceites vírgenes valores máximos de $15 \mathrm{meqO}_{2} / \mathrm{Kg}$, y $5 \mathrm{meqO}_{2} / \mathrm{Kg}$ respectivamente. El índice de yodo constituye una medida del grado de insaturación del aceite, característica muy importante que está relacionada con el punto de fusión del mismo, de tal manera que a mayor cantidad de insaturación el punto de fusión del aceite será menor. Los valores promedios y la desviación estándar $(\bar{X} \pm \sigma)$ del aceite extraído por prensado y por soxhlet son de: $85.1 \pm 0.7 \mathrm{cg} \mathrm{l} / \mathrm{g}$ y $83.0 \pm 0.7 \mathrm{cg} \mathrm{l} / \mathrm{g}$ respectivamente; estos valores se encuentran dentro del rango establecido en las normas CODEX y ANDI con valores máximos entre $75-94 \mathrm{cg} \mathrm{l} / \mathrm{g}$ reglamentados para aceites en la industria alimenticia. De acuerdo al índice de yodo, los aceites en general se pueden clasificar en: secantes con valores entre 140 y $210 \mathrm{cg} \mathrm{l} / \mathrm{g}$, semisecantes entre 100 y $140 \mathrm{cg} \mathrm{l/g}$ y no secantes por debajo de $100 \mathrm{cg} \mathrm{l/g}$; dependiendo de esta clasificación, el aceite puede tener diferentes usos en la industria; el índice de yodo presentado por el aceite de la almendra de marañón se encuentra en el rango de los no secantes, razón por el cual puede ser utilizado en la elaboración de cremas y jabones líquidos. El índice de saponificación para el aceite extraído por prensado es de $194.4 \pm 0.9 \mathrm{mgKOH} / \mathrm{g}$ y el de soxhlet $175.3977 \pm 0.4010 \mathrm{mgKOH} / \mathrm{g}$, siendo mayor este valor para el obtenido por el primer método, lo que indica mayor presencia de ácidos grasos de bajo peso molecular, porque los esteres de estos ácidos grasos requieren mayor cantidad de álcali para la saponificación haciéndolos mucho mas olorosos.

El índice de saponificación para el aceite obtenido por el metodo de prensado sugiere su posible uso en la industria de jabones y cosméticos, en la cual se exige un valor mínimo de $185 \mathrm{mgKOH} / \mathrm{g}$; de igual forma cumple con las normas CODEX y ANDI las cuales establecen un rango entre (184- 
196) $\mathrm{mgKOH} / \mathrm{g}$, facilitando su uso en la industria alimenticia; a diferencia del aceite extraído por soxhlet el cual presentó un índice de saponificación menor al reglamentado por las normas de la industria alimenticia.

Para determinar la composición porcentual de ácidos grasos en el aceite extraído por soxhlet de la almendra del marañón, se hizo un barrido de seis a veinte átomos de carbono, comprendiendo los tiempos de retención de 4 a 23 minutos, obteniéndose un $81.36 \%$ de ácidos grasos insaturados, formado por el $61.36 \%$ del ácido oleico (monoinsaturado), el $19.48 \%$ del ácido linoleico y el $0.52 \%$ del ácido linolénico (poliinsaturados). El $18.64 \%$ de ácidos grasos obtenidos fueron saturados formado por ácido palmítico con $10.43 \%$ y el ácido esteárico con $8.21 \%$, véase tabla 4 , estos resultados obtenidos del aceite extraído del marañon, cultivado en la costa norte de Colombia, son comparables con otro estudios realizados al aceite de la almendra del marañon cultivado en Pakistan a diferentes condiciones ambientales (Sini y Nair, 2009).

Tabla 4: Composición porcentual de ácidos grasos en el aceite de la almendra de Marañón (Anacardium occidentale $L$ )

\begin{tabular}{|c|c|c|c|}
\hline Ácidos grasos & Nombre Común & $\begin{array}{l}\text { Tiempo de retención } \\
\text { (min) }\end{array}$ & Acido graso ( $\%$ ) \\
\hline C $6: 0$ & Caproico & 4.250 & 0.00 \\
\hline C 8:0 & Caprilico & 4.500 & 0.00 \\
\hline C 10:0 & Cáprico & 5.250 & 0.00 \\
\hline C 12:0 & Laurico & 6.600 & 0.00 \\
\hline C 14:0 & Miristico & 8.550 & 0.00 \\
\hline C 16:0 & Palmitico & 11.338 & 10.43 \\
\hline C $16: 1$ & Palmitoleico & 13.000 & 0.00 \\
\hline C 18:0 & Estearico & 14.933 & 8.21 \\
\hline C 18:1 & Oleico & 15.802 & 61.36 \\
\hline C $18: 1$ trans & Elaidico & 16.000 & 0.00 \\
\hline C $18: 2$ & Linoleico & 17.072 & 19.48 \\
\hline C $18: 3$ & Linolenico & 18.308 & 0.52 \\
\hline C 20:0 & Araquidico & 22.300 & 0.00 \\
\hline
\end{tabular}

\section{CONCLUSIONES}

El método que presentó mejor rendimiento para la extracción del aceite de la almendra de Anacardium occidentale $L$, es el de soxhlet utilizando como solvente el hexano. Los aceites obtenidos por prensado y soxhlet poseen buenas características organolépticas y excelentes propiedades fisicoquímicas, que cumple con las normas internacionales para aceites utilizados en la industria de jabones y cosméticos.

De acuerdo con los valores obtenidos en las propiedades fisicoquímicas y el alto contenido de ácido oleico (61.36\%), siendo este un ácido graso esencial (omega 9 ) se puede decir que el aceite extraído por el método de prensado podría ser utilizado en la industria alimenticia, pero el de soxhlet no es el mejor para fines comestibles.

\section{AGRADECIMIENTOS}

Al centro de investigaciones de la Universidad de Córdoba por el financiamiento de este proyecto y a la empresa SGS, Colombia. S.A.

\section{REFERENCIAS}

AOCS Ca 5a-40. Official Method Free Fatty Acids, Sampling and Analysis of Commercial Fats and Oils. Copyright The American Oils Chemist's Society. Urbana - Illinois. USA (1997). 
AOCS Cd 1-25. Official Method lodine Value of Fats and Oils Wijs Method, Sampling and Analysis of Commercial Fats and Oils. Copyright The American Oils Chemist's Society. Urbana - Illinois. USA (1997)

AOCS Cd 3-25. Official Method Saponification Value, Sampling and Analysis of Commercial Fats and Oils. Copyright The American Oils Chemist's Society. Urbana - Illinois. USA (2003).

AOCS Cd 8-53. Official Method Peroxide Value Acetic Acid (Chloroform Method), Sampling and Analysis of Commercial Fats and Oils. Copyright The American Oils Chemist's Society. Urbana Illinois. USA (2003).

AOCS Ce 1C-89. Official Method Fatty Acid Composition by GC: cis, cis and trans isomers. Copyright The American Oils Chemist's Society. Urbana - Illinois. USA (1992).

Belén, D.J., I .López, M. González., M. J Moreno., C.Medina, Evaluacion fisicoquímica de la semilla y del aceite de corozo (Acrocomia aculeata Jac), Grasas y Aceites: 56, 311-316 (2005).

Cenzano I, Métodos Oficiales de Análisis de Alimentos. AMU Ediciones -Mundi Prensa Libros S.A. 201-202, España, Madrid, (1994).

Chipojola F.M., W. Mwase., M. B. Kwapata., J. M. Bokosi., J. P. Njoloma., M. F. Malir, Morphological characterization of cashew (Anacardium occidentale L.) in four populations in Malaw, African Journal Biotechnology: 8, 5173-5181 (2009).

FAO / OMS. CODEX Stan 19-1981, Norma del Codex para Grasas y Aceites Comestibles No Regulados por Normas Individuales, Codex Alimentarius Official Standards, Canadá (1999).

FAO / OMS. Codex Stan 19-1981, Norma delCodex para los Aceites de Oliva y Aceites de Orujo de Oliva. Codex Alimentarius Official Standards. Canadá (2003).

Hernández, C., A. Mieres., Z. Niño., S.Pérez, Efecto de la refinación física sobre el aceite de la almendra del corozo (Acrocomia aculeata,. Información tecnológica: 18, 59-68 (2007).

Kubo J., J. Lee., I.Kubo, Anti-Helicobacter Pylori Agents from the Cashew Apple, Journal of Agricultural and Food Chemistry: 47, 533-537 (1999).

Mestres L.M., J.M. Souquet., H.Fulcrand., C. Bouchut., M. Reines., J.M. Brillouet, Monomeric phenols of cashew apple (Anacardium occidentale L), Food Chemistry: 112, 851-857 (2009).

Ofusori, D.A. y otros seis autores, Microestructural study of the effect of ethanolic extract of Cashew stem bark Anacardium occidentale on the brain and Kidney of Swiss albino mice, Internet Journal of Alternative medicine: 5, 1-3 (2008).

Omosuli, S.V., A. Ibrahim., D. Oloye., R. Agbaje., B. Jude, Proximate and mineral composition of roasted and defatted cashew nut (Anacardium occidentale) flour, Pakistan Journal of nutrition: 8 , 1649-1651 (2009).

Páez M.S., C.M. Romero., D. Pérez, A comparative study of the volumetric properties of dilute aqueous solutions of 1-propanol, 1,2-propanediol, 1,3-propanediol, and 1,2,3-propanetriol at various temperatures, J. Chem. Thermodynamics: 40, 1645-1653 (2008).

Páez M.S., J.Lafont., A. Portacio, Efecto de la temperatura sobre la viscosidad de soluciones acuosas diluidas de 1,2-pentanodio, Información tecnológica: 20, 55-60 (2009).

Santoa R, Production and Characterization of the Cashew (Anacardium occcidentale L.) Peduncle Bagasse Ashes, Journal of Food Engineering: 79, 1432-1437 (2007).

Setianto W.B., S.Yoshikawa., R. L. Smith., H. Inomata., L. J. Florusse., C.J. Peters, Pressure profile separation of phenolic liquid compound from cashew (Anacardium occidentale $L$ )shell with 
supercritical carbon dioxide and aspect of its phase equilibria, J. of supercritical fluids: 48, 203-210 (2009).

Sini. A., C.K. Nair, Quality of oil from edible cashew nuts and cashew kernel processing rejects, Journal of Food Science and Technology:46, 163-165 (2009).

Trevejo E y M.Maury, Extracción y Caracterización del Aceite de Poraqueiba serícea tulasne (UMARI,. Revista Amazónica de Investigación Alimentaria: 2, 1-18 (2002). 